\title{
Tu 0108
}

Outcropping Analogs and Multiscale Fracture Patterns in the Jandaíra Formation

\author{
G. Bertotti* (Delft University of Technology), F.H. Bezerra (Universidade \\ Federal do Rio Grande do Norte), K. Bisdom (Delft University of \\ Technology), C. Cazarin (Petrobras) \& J. Reijmer (VU University \\ Amsterdam)
}

\section{SUMMARY}

Outcropping analogs can provide key information on the 3D organization of fracture networks affecting carbonate reservoirs. Such information, however, needs to be integrated in a consistent work flow which includes i) 3D geometric model of the reservoir architecture, ii) mechanic modeling to determine areas of fracturing, iii) characterization of fracture networks, iv) determination of fracture-related permeability in small representative volumes and $v$ ) fluid flow simulations.

Fracture networks have been recently analysed from km-scale pavements of the Jandaira Formation in NE Brazil. Combining images from a drone with outcrop analysis and thin section we present the first multiscale characterization of the fracture field affecting the shallow water carbonates. 


\section{Introduction}

Predicting fracture-related permeability in carbonate reservoirs is a major challenge for hydrocarbon Industry. Not only the architecture of fracture network can vary in space (from one lithology to the other) and in time (in relations with diagenesis and subsidence), but (aggressive) fluids circulating through fractures can provoke dissolution and possibly karstification. Indeed, all these features are present in Brazilian carbonate reservoirs.

A consistent work-flow has been designed to achieve relevant predictions of fracture related permeability in reservoirs (Fig. 1). The work-flow consists of i) building a geometric model of the reservoir, ii) finite element modelling work to determine the distribution of fractured domains and the orientation of expected fractures, iii) outcrop studies to predict geometric properties of fracture networks, iv) permeability prediction in the various parts of the reservoir and, v) fast flow simulations to quantify the sensitivity of results to the various parameters.
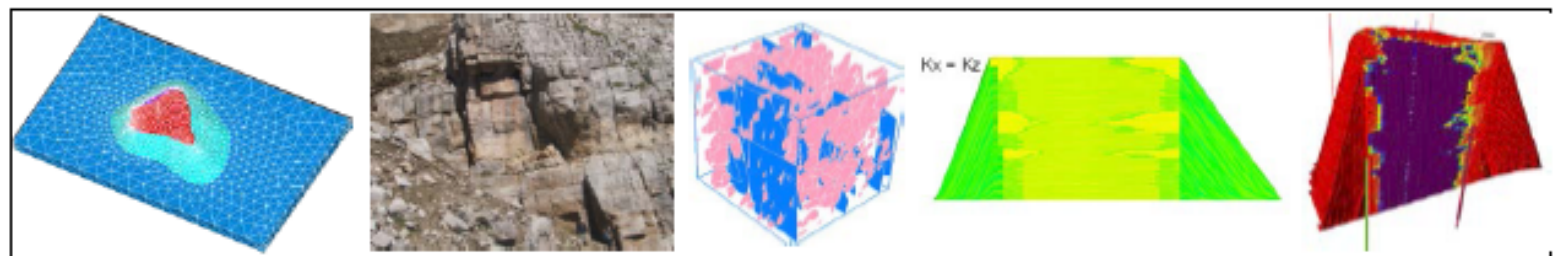

Fig. 1 The work flow. From left to right: geometric and mechanic model, outcrop-based characterization of fracture networks, discrete fracture network models, platform-scale permeability and flow experiments

Outcrop studies are key in predicting the 3D architecture networks, that is, the dimensions of fractures, their spatial arrangement and relations with bedding surfaces.

Deriving the architecture of fracture networks critically hinges on our ability to acquire complete data sets from outcrops honouring their multiscale character evident from field and subsurface observations. Acquisition of this data sets presents major challenges, especially in large pavements, such as the ones we investigate in this study.

\section{Data acquisition}

In the frame of the Petrobras-supported Porocarste project we have acquired fracture data from $5 \mathrm{~km}$ sized pavements of the Jandaira Formation (Apodi, RN). To capture large-scale faults and fractures down to the size of $\mathrm{dm}$, we have used a microdrone recently acquired by the Delft University of Technology with the financial support of the Netherlands Research School on Solid Earth Studies (ISES) (Fig. 2a). Flying at heights of $60-70 \mathrm{~m}$ along a pre-given grid, the drone has acquired digital images of the pavements with a $70 \%$ frontal and $40 \%$ lateral overlap (Fig. $2 b$ ).

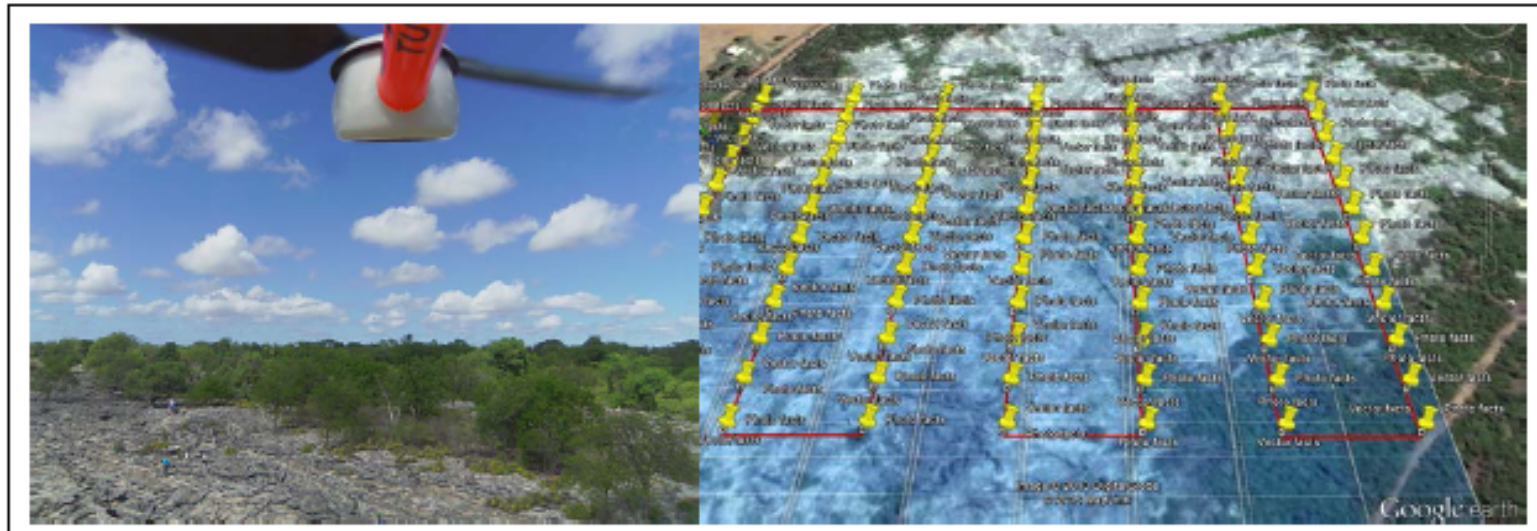

Fig. 2 Air-borne data acquisition. a) view from drone; b) an example of a grid flown by the dro 
Using commercial software, the images have been immediately stitched and translated in enriched Digital Elevation Models, that is, $\mathrm{x}, \mathrm{y}, \mathrm{z}$ surfaces on which photographic images have been draped. Having the images stitched at "base camp" was crucial to allow a quality check, early data and steer acquisition during the coming days.

To interpret fractures observed from the airborne device and refine the larger-scale observations we have examined 15 structural stations in the pavements covered by the drone. Stylolites (both parallel and perpendicular to bedding), veins and shear fractures have been characterized and samples have been taken using a drilling machine.

\section{Preliminary results}

The data acquired during the 2013 campaign are being processed and the main results will be available for the time of the Workshop. The very preliminary interpretation of our data suggest that: - The carbonates of the Jandaira formation are affected a few sub-vertical fault zones typically formed reactivating joint systems.

- Intervening blocks are traversed by a large amount of fractures. In images from the drone they all appear as open fractures resulting predominantly from dissolution. Interestingly, different domains display different dominant fracture geometries

- Inspection at the outcrop scale shows that these open fractures were steep stylolites, veins or, less commonly, conjugate systems of shear fractures (Fig. 3). From our preliminary interpretation it seems that most of these features are compatible with N-S compression.

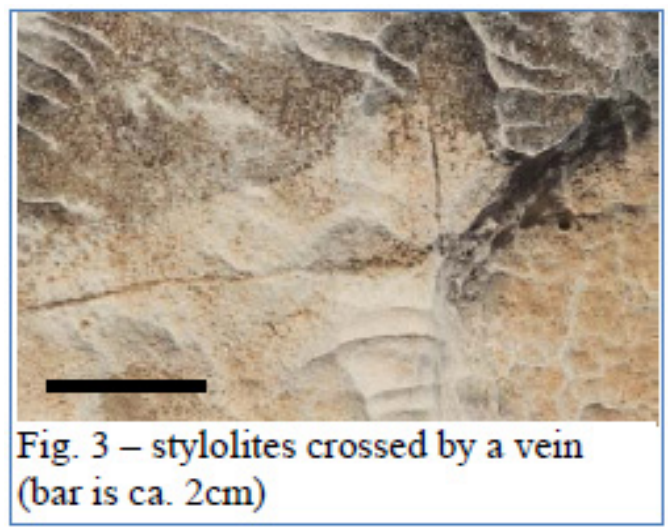

\section{Considerations and future work}

The multidisciplinary character of the acquisition we have performed has proven to be successful and has led to a large amount of data which to be processed in the near future. Five projects are defined

1) Geometric analysis of fracture patterns from drone images. Fractures will be traced on the backdrop of the stitched and georeferenced images and statistically analysed for their orientation, length, abutment relations and spatial density parameters. It will be followed by the construction of multiscale Discrete Fracture Network models used to determine permeabilities in the different domain of the outcrop.

2) Fractures and caves. Maps with the results of the fracture analysis will be compared with maps of cave and karts distribution to infer links between fractures and dissolution patterns.

3) Sample scale. Sample-scale observation will lead to a better understanding of the processes of stylolitization (related both to overburden en tectonics) and of vein formation. Polished sections and thin section are presently being obtained from the samples to reconstruct the evolution of porosity, of cement in the primary rock as well as in the veins.

4) Thin sections will be used to analyse the cements in the bed-rock and, more importantly in the veins. Combining CL studies and fluid inclusion analysis we will derive relevant information on fluids circulating through the carbonates of the Jandaira formation as well as on physical parameters such as pressure and temperature.

\section{Acknowledgements}

This generous support of Petrobras is gratefully acknowledged. All people participating in the field campaign are thanked: M. Vieira, F. Hameka, A. Vis, C. Paulides, M. van Eijk, N. Srivastava, S. Vieira, J. Rebelo and F.C. Nogueira. N. Hardebol (Delft) is acknowledged for his constant help and discussions. 